\title{
Crescimento e conteúdo de prolina em plântulas de guandu submetidas a estresse osmótico e à putrescina exógena
}

\author{
Juliana Geseíra Monteiro(1), Flávio José Rodrigues Cruz(1), Mariana Batista Nardin(1) \\ e Durvalina Maria Mathias dos Santos ${ }^{(1)}$
}

\begin{abstract}
(1)Universidade Estadual Paulista, Departamento de Biologia Aplicada à Agropecuária, Via de Acesso Prof. Paulo Donato Castellane, s/no, CEP 14884900 Jaboticabal, SP, Brasil. Email: geseira@hotmail.com, fjrc@bol.com.br, mariana.nbatista@yahoo.com.br, dumaria@fcav.unesp.br
\end{abstract}

\begin{abstract}
Resumo - O objetivo deste trabalho foi verificar o efeito da aplicação da poliamina putrescina no acúmulo de prolina e no crescimento inicial de plântulas de guandu cultivadas sob estresse hídrico ou salino, e avaliar se esta poliamina pode ser usada como atenuadora desses estresses. Sementes de duas cultivares de guandu, BRS Mandarim e Caqui, foram germinadas em caixas gerbox forradas com papel de filtro umedecido com $0,0,-0,2$, $-0,3,-0,4,-0,5 \mathrm{MPa}$ de PEG 6000 ou $0,20,40,60,80$ e $120 \mathrm{mmol} \mathrm{L}^{-1}$ de $\mathrm{NaCl}$, na ausência $\left(0 \mathrm{mmol} \mathrm{L}{ }^{-1}\right)$ ou na presença $\left(0,5 \mathrm{mmol} \mathrm{L}^{-1}\right)$ de putrescina. Utilizou-se o delineamento inteiramente casualizado, em arranjo fatorial $2 \times 5 \times 2$ e $2 \times 6 \times 2$ (duas cultivares, cinco níveis de deficiência hídrica ou seis níveis de estresse salino, e presença ou ausência de putrescina), com quatro repetições. $\mathrm{O}$ crescimento das plântulas é acentuadamente reduzido pelas condições de restrição hídrica e salinidade. A putrescina exógena incrementa, sob ambos os estresses, os teores de prolina nas plântulas de guandu, especialmente na cultivar BRS Mandarin, e atenua os efeitos da deficiência hídrica moderada nas cultivares estudadas. Os estresses de restrição hídrica e de salinidade causam acúmulo de prolina na parte aérea e nas raízes de ambas as cultivares. A prolina pode ser considerada um bom indicador bioquímico e fisiológico desses estresses em plântulas de guandu.
\end{abstract}

Termos para indexação: Cajanus cajan, indicador bioquímico, poliamina, restrição hídrica, salinidade, solutos compatíveis.

\section{Growth and proline content in pigeon pea seedlings subjected to osmotic stress and to exogenous putrescine}

\begin{abstract}
The objective of this work was to verify the effect of the application of the polyamine putrescine on the accumulation of proline and on the early seedling growth of pigeon pea subjected to water or salt stress, and to evaluate whether this polyamine can be used to alleviate these stresses. Seeds of two cultivars of pigeon pea, BRS Mandarim and Caqui, were germinated in gerbox boxes lined with filter papers soaked with $0.0,-0.2,-0.3,-0.4,-0.5 \mathrm{MPa}$ PEG 6000 or $0,20,40,60,80$, and $120 \mathrm{mmol} \mathrm{L}^{-1} \mathrm{NaCl}$, in the absence $\left(0 \mathrm{mmol} \mathrm{L}^{-1}\right)$ or presence $\left(0.5 \mathrm{mmol} \mathrm{L}^{-1}\right)$ of putrescine. A completely randomized design was used, in a $2 \times 5 \times 2$ and $2 \times 6 \times 2$ factorial arrangement (two cultivars, five levels of drought or six levels of salt stress, and presence or absence of putrescine), with four replicates. Seedling growth is highly reduced by water restriction and salinity conditions. Exogenous putrescine increases, under both stresses, the proline contents in pigeon pea seedlings, especially in the cultivar BRS Mandarin, and alleviates the effects of moderate water deficiency on the studied cultivars. Water restriction and salinity stresses cause accumulation of proline content in shoots and roots of both cultivars. Proline can be considered a good biochemical and physiological indicator of these stresses in pigeon pea seedlings.
\end{abstract}

Index terms: Cajanus cajan, biochemical indicator, polyamine, water restriction, salinity, compatible solutes.

\section{Introdução}

Os estresses abióticos em plantas têm despertado grande interesse da pesquisa, que tem buscado incessantemente por cultivares mais produtivas sob condições desfavoráveis de cultivo. Os estresses decorrentes da deficiência hídrica e da salinidade do solo são os mais estudados, em razão de seu forte impacto sobre o crescimento e a produtividade das plantas. A restrição hídrica é responsável por sérias disfunções nas plantas, pelo acentuado decréscimo que causa no turgor das células, imprescindível para

Pesq. agropec. bras., Brasília, v.49, n.1, p.18-25, jan. 2014

DOI: 10.1590/S0100-204X2014000100003 
o adequado metabolismo celular. Já o estresse salino pode ser responsável por alterações morfológicas, fisiológicas e bioquímicas, com reflexos sobre o transporte de água e nutrientes nas plantas (Munns, 2011; Filippou et al., 2014).

Estresses abióticos são considerados os que mais limitam a produção de culturas agrícolas ao redor do mundo (Munns, 2011; Filippou et al., 2014). Wilhelm (2014) apresenta diversos estudos sobre o assunto. No entanto, pesquisas que tratam do tema ainda são necessárias para maior compreensão dos mecanismos de adaptação das plantas a ambientes com deficit hídrico e aos que apresentam salinização grave (Kumar et al, 2011; Munns, 2011; Fariduddin et al., 2013).

Vários mecanismos de proteção são ativados nas plantas em resposta a condições adversas de crescimento. O ajuste osmótico constitui-se um dos mecanismos fisiológicos mais eficazes para manutenção da turgescência celular, sob condições de baixo potencial hídrico no solo (Marijuan \& Bosch, 2013). Ele confere proteção por curtos períodos de tempo de estresse e é importante quando as condições não tardam a se normalizar. Esse mecanismo se estabelece mediante o acúmulo, no vacúolo ou no citosol, de solutos compatíveis (prolina, glicina betaína, trealose, sacarose, poliaminas, manitol, pinitol, entre outros) que contribuem para a manutenção do equilíbrio hídrico e a preservação da integridade de proteínas, enzimas e membranas celulares (Ashraf et al., 2011; Marijuan \& Bosch, 2013). Esses solutos também têm função osmoprotetora contra subprodutos tóxicos do metabolismo, resultantes do estresse hídrico. $\mathrm{O}$ acúmulo de solutos compatíveis não é nocivo ao metabolismo celular e, por aumentar a pressão osmótica no interior das células, mantêm a absorção de água e a pressão de turgor das células, o que concorre para a continuidade dos processos fisiológicos, ainda que em níveis menores (Marijuan \& Bosch, 2013).

O aminoácido prolina é o soluto compatível mais estudado, em razão de sua sensibilidade de resposta a condições de estresse (Trovato et al., 2008; Verbruggen \& Hermans, 2008; Ashraf et al., 2011). Em plantas sob estresse, o conteúdo de prolina pode aumentar até 100 vezes, em comparação ao observado em plantas cultivadas sob condições normais (Verbruggen \& Hermans, 2008). Esse acúmulo fornece um importante parâmetro para a seleção de plantas resistentes (Verbruggen \& Hermans, 2008). Além disso, é comum a constatação de que teores aumentados de prolina atenuam os efeitos do estresse hídrico (Cvikrová et al., 2013; Filippou et al., 2014). Segundo os autores, o acúmulo ocorre pela síntese "de novo" ou pela inibição do processo de oxidação da prolina. Embora haja abundante literatura sobre o acúmulo de prolina, em plantas sob vários tipos de estresse, não se sabe ao certo se esse acúmulo sempre ocorre (Trovato et al., 2008; Ashraf et al., 2011; Szábados et al., 2011).

As poliaminas (putrescina, espermidina, espermina e cadaverina) ocorrem naturalmente em plantas. Estas são substâncias de baixo peso molecular, policatiônicas, alifáticas e nitrogenadas (Takahashi \& Kakehi, 2010; Hussain et al., 2011; Fariduddin et al., 2013), que podem ser utilizadas por algumas plantas na defesa contra condições adversas. O interesse pelas poliamidas se deve ao fato de elas apresentarem vias metabólicas interconectadas com diferentes rotas envolvidas na formação de moléculas e metabólitos relevantes para sinalizar as respostas das plantas ao estresse (Duan et al., 2008; Takahashi \& Kakehi, 2010; Fariduddin et al., 2013; Gupta et al., 2013; Filippou et al., 2014). Trabalhos recentes destacam que as poliaminas estão envolvidas na proteção das plantas à restrição hídrica e à salinidade (Takahashi \& Kakehi, 2010; Hussain et al., 2011; Gupta et al., 2013; Filippou et al., 2014; Kotakis et al., 2014). Os relatos indicam que, sob condições de estresse, o conteúdo de poliaminas endógenas em algumas plantas podem diminuir. Dessa forma, o uso de poliaminas exógenas, em especial da putrescina, pode atenuar os efeitos de vários tipos de estresse nas plantas e aumentar a tolerância das culturas (Quinet et al., 2010; Ashraf et al., 2011; Hussain et al., 2011; Sharma et al., 2011; Amri \& Mohammadi, 2012; Gupta et al., 2013; Kotakis et al., 2014).

Diversos autores observaram estreita relação entre o acúmulo de prolina e de poliaminas em plantas sob estresse, pelo fato de elas compartilharem o mesmo precursor, a ornitina, nas rotas de biossíntese (Alcázar et al., 2010; Quinet et al., 2010; Filippou et al., 2013, 2014; Lutts et al., 2013; Kotakis et al., 2014). Portanto, é relevante averiguar a possiblidade de a putrescina melhorar as respostas de plantas sob estresse abiótico, mediante a avaliação do crescimento e a determinação dos teores de prolina.

O objetivo deste trabalho foi verificar o efeito da aplicação da poliamina putrescina no acúmulo de prolina e no crescimento inicial de plântulas de guandu 
cultivadas sob estresse hídrico ou salino, e avaliar se esta poliamina pode ser usada como atenuadora desses estresses.

\section{Material e Métodos}

O experimento foi realizado no laboratório de Fisiologia Vegetal da Faculdade de Ciências Agrárias e Veterinária, da Universidade Estadual Paulista, em Jaboticabal, SP. Foram utilizadas sementes selecionadas de guandu [Cajanus cajan (L.) Millsp.], das cultivares BRS Mandarim e Caqui, cedidas pela empresa Wolf Seeds do Brasil, Ribeirão Preto, SP.

$\mathrm{O}$ estudo foi realizado em dois experimentos, cada um conduzido em delineamento inteiramente casualizado, em arranjo fatorial $(2 \times 5 \times 2$ ou $2 \times 6 \times 2)$ constituído por: duas cultivares de guandu; cinco tratamentos de restrição hídrica $(0,0,-0,2,-0,3$, $-0,4$ e $-0,5 \mathrm{MPa}$ ) simulada por PEG 6000 (primeiro experimento) ou seis tratamentos salinos $(0,20,40,60$, 80 e $120 \mathrm{mmol} \mathrm{L}^{-1}$ de $\mathrm{NaCl}$; segundo experimento); e duas concentrações da poliamina putrescina $(0 \mathrm{e}$ 0,5 $\mathrm{mmol} \mathrm{L}^{-1}$ ) (SigmaAldrich, St. Louis, MO, EUA), conforme recomendações de Jiang et al. (2012). Foram utilizadas quatro repetições em ambos os experimentos, com 25 sementes por repetição.

A semeadura foi realizada em caixas de plástico tipo gerbox esterilizadas, contendo papel de filtro umedecido com as soluções de PEG 6000 ou NaCl, na presença ou na ausência da putrescina. As caixas de plástico foram mantidas em câmara de germinação, a $25^{\circ} \mathrm{C} \pm 0,2$ e fotoperíodo de 12 horas.

No décimo dia após a semeadura, foram avaliados os teores de prolina e o crescimento inicial da parte aérea e das raízes primárias. A prolina livre da parte aérea e das raízes foi extraída pelo método do ácido sulfossalicílico e determinada em espectrofotômetro, a $520 \mathrm{~nm}$, de acordo com Bates et al. (1973). A massa de matéria seca das partes vegetativas foi avaliada após secagem em estufa de ventilação forçada de ar, a $60^{\circ} \mathrm{C}$, por 48 horas.

Regressões polinomiais foram ajustadas aos dados quantitativos referentes aos tratamentos de restrição hídrica e salinidade, e o teste de Tukey, a 5\% de probabilidade, foi utilizado para comparação dos parâmetros qualitativos, relacionados à presença ou à ausência de putrescina e às duas cultivares de guandu.

\section{Resultados e Discussão}

A deficiência hídrica foi o estresse com maior impacto nos teores de prolina e no crescimento das plântulas; porém, os efeitos do estresse salino sobre essas variáveis também foram significativos (Tabela 1). Os efeitos da deficiência hídrica sofreram interação de todos os demais fatores, enquanto os do estresse salino somente interagiram com as cultivares.

Os teores de prolina da parte aérea e da raiz aumentaram com a redução da disponibilidade hídrica ou com o aumento da salinidade (Figuras 1 e 2) e, nas raízes da cultivar BRS Mandarim, foram especialmente sensíveis à salinidade, principalmente a partir da concentração salina de $20 \mathrm{mmol} \mathrm{L}^{-1}$ (Figura 2).

$\mathrm{O}$ crescimento da parte aérea e das raízes foi negativamente afetado pelos estresses, em ambas as cultivares (Figuras 1 e 2). A redução no crescimento das plântulas de guandu pelo aumento da salinidade foi expressivo, mesmo com o incremento observado nos teores de prolina, na parte aérea e nas raízes. A redução do potencial hídrico, induzida pelo PEG ou pelo sal, bem como os efeitos citotóxicos dos íons $\mathrm{Na}^{+}$e $\mathrm{Cl}^{-}$, no caso da salinidade, são responsáveis pela redução no crescimento das plântulas (Gordin et al., 2012; Maia et al., 2012).

Em situações de restrição hídrica ou de toxidez por $\mathrm{Na}^{+}$e $\mathrm{Cl}^{-}$, a degradação de compostos celulares e a síntese de metabólitos indesejáveis podem promover redução drástica do crescimento (Machado Neto et al., 2006). Portanto, os níveis de salinidade foram efetivos em reduzir o crescimento das plântulas de guandu, apesar do acúmulo de prolina na matéria seca. Filippou et al. (2014), no entanto, relatam que o acúmulo desse aminoácido está envolvido na indução de tolerância a estresses osmóticos severos e é uma das estratégias de sobrevivência adotadas pela espécie Ailanthus altissima, quando submetida a estresses salinos de 150 e $300 \mathrm{mmol} \mathrm{L}^{-1}$.

O maior acúmulo de prolina nas raízes da cultivar BRS Mandarim não foi capaz de proteger o crescimento destas, em comparação à cultivar Caqui (Figura 1).

Com o desdobramento da interação entre os efeitos de PEG e putrescina exógena sobre o acúmulo de prolina, constatou-se que, em níveis moderados de restrição hídrica, a putrescina favoreceu o acúmulo do aminoácido na parte aérea (Figura 3), sem efeito expressivo sobre a massa de matéria seca, tanto da parte aérea como da raiz. 
Tabela 1. Quadrados médios dos teores de prolina e do crescimento inicial de plântulas de guandu (Cajanus cajan) submetidas aos estresses hídrico (PEG 6000) ou salino ( $\mathrm{NaCl}$ ), com e sem adição de putrescina.

\begin{tabular}{|c|c|c|c|c|c|}
\hline \multirow[t]{2}{*}{ Fonte de variação } & \multirow[t]{2}{*}{ GL } & \multicolumn{2}{|c|}{ Teor de prolina ( $\mu \mathrm{mol} \mathrm{g}^{1}$ da matéria fresca) } & \multicolumn{2}{|c|}{ Crescimento inicial (g por plântula) } \\
\hline & & Parte aérea & Raízes primárias & Parte aérea & Raízes primárias \\
\hline & \multicolumn{5}{|c|}{ Experimento com restrição hídrica (PEG 6000) } \\
\hline Cutivares (C) & 1 & $0,4526 * *$ & $0,0240^{\text {ns }}$ & $0,0020 * *$ & $0,0234 * *$ \\
\hline PEG $(\mathrm{H})$ & 4 & $33,0169 * *$ & $38,0061 * *$ & $0,0982 * *$ & $0,0185^{* *}$ \\
\hline Putrescina $(\mathrm{P})$ & 1 & $0,4702 * *$ & $0,2827 * *$ & $0,0016^{* *}$ & $0,0004^{\mathrm{ns}}$ \\
\hline Interação (CxH) & 4 & $1,0234 * *$ & $0,4930 * *$ & $0,0011 * *$ & $0,0012 * *$ \\
\hline Interação (CxP) & 1 & $8,4065 * *$ & $4,2549 * *$ & $0,0036^{* *}$ & $0,0098^{\mathrm{ns}}$ \\
\hline Interação (HxP) & 4 & $1,1935 * *$ & $0,9392 * *$ & $0,0006^{* *}$ & $0,0026^{* *}$ \\
\hline Interação (CxHxP) & 4 & $0,7370 * *$ & $0,9069 * *$ & $0,0007 * *$ & $0,0007^{*}$ \\
\hline Resíduo & 60 & 0,0053 & 0,0111 & 0,0001 & 0,0003 \\
\hline \multirow[t]{2}{*}{$\mathrm{CV}(\%)$} & & 3,5246 & 3,6180 & 8,0671 & 12,4579 \\
\hline & \multicolumn{5}{|c|}{ Experimento com estresse salino $(\mathrm{NaCl})$} \\
\hline Cultivares (C) & 1 & $371,8371 * *$ & $11950,5532 * *$ & $0,0279 * *$ & $0,0007 * *$ \\
\hline Estresse salino $(\mathrm{S})$ & 5 & $24168,1390 * *$ & $33019,0123 * *$ & $0,0180 * *$ & $0,0074 * *$ \\
\hline Putrescina (P) & 1 & $2333,7368^{* *}$ & $7615,7030^{* *}$ & $0,0058 * *$ & $0,0031 * *$ \\
\hline Interação (CxS) & 5 & $453,8574 * *$ & $3440,6659^{* *}$ & $0,0084 * *$ & $0,0006^{* *}$ \\
\hline Interação (CxP) & 1 & $1,3084^{\mathrm{ns}}$ & $45,0628^{\mathrm{ns}}$ & $0,0000^{\mathrm{ns}}$ & $0,0013 * *$ \\
\hline Interação (SxP) & 5 & $84,4373^{\mathrm{ns}}$ & $297,3983^{\text {ns }}$ & $0,0017^{*}$ & $0,0005^{* *}$ \\
\hline Interação (CxSxP) & 5 & $46,6774^{\mathrm{ns}}$ & $378,2510^{\mathrm{ns}}$ & $0,0041 * *$ & $0,0005^{*}$ \\
\hline Resíduo & 72 & 42,0388 & 202,1998 & 0,0006 & 0,0010 \\
\hline CV (\%) & & 6,4220 & 10,6403 & 12,8301 & 8,4279 \\
\hline
\end{tabular}

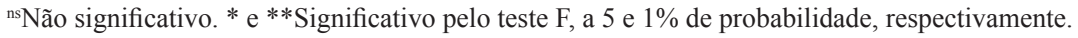
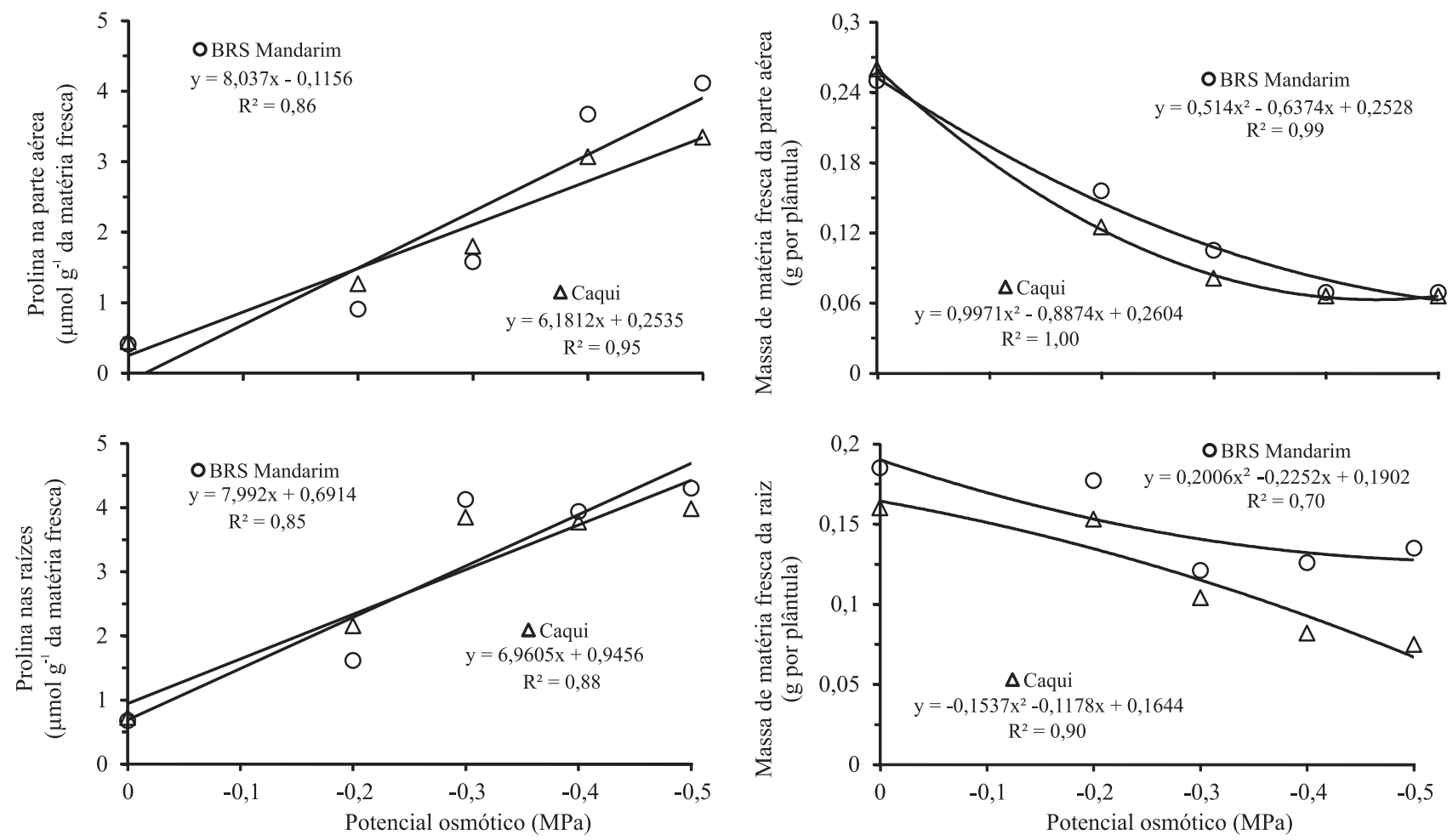

Figura 1. Teores de prolina ( $\mu \mathrm{mol} \mathrm{g}{ }^{-1}$ da matéria fresca) e crescimento inicial ( $\mathrm{g}$ por plântula) da parte aérea e de raízes primárias de guandu (Cajanus cajan), das cultivares BRS Mandarim e Caqui, submetidas à deficiência hídrica (PEG 6000). 

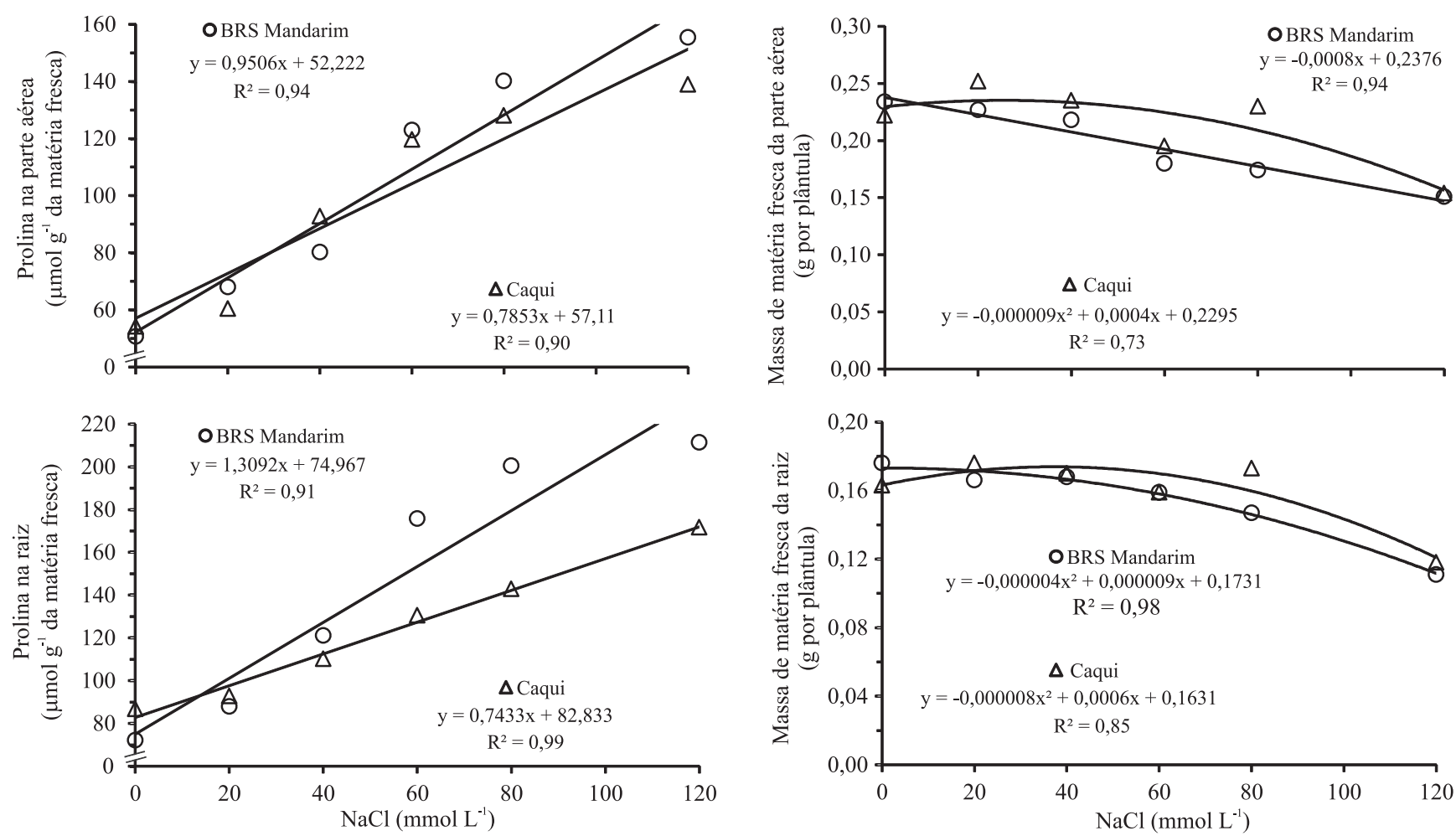

Figura 2. Teores de prolina ( $\mu \mathrm{mol} \mathrm{\textrm {g } ^ { - 1 }}$ da matéria fresca) e crescimento inicial (g por plântula) da parte aérea e de raízes primárias de guandu (Cajanus cajan), das cultivares BRS Mandarim e Caqui, submetidas ao estresse salino ( $\mathrm{NaCl})$.
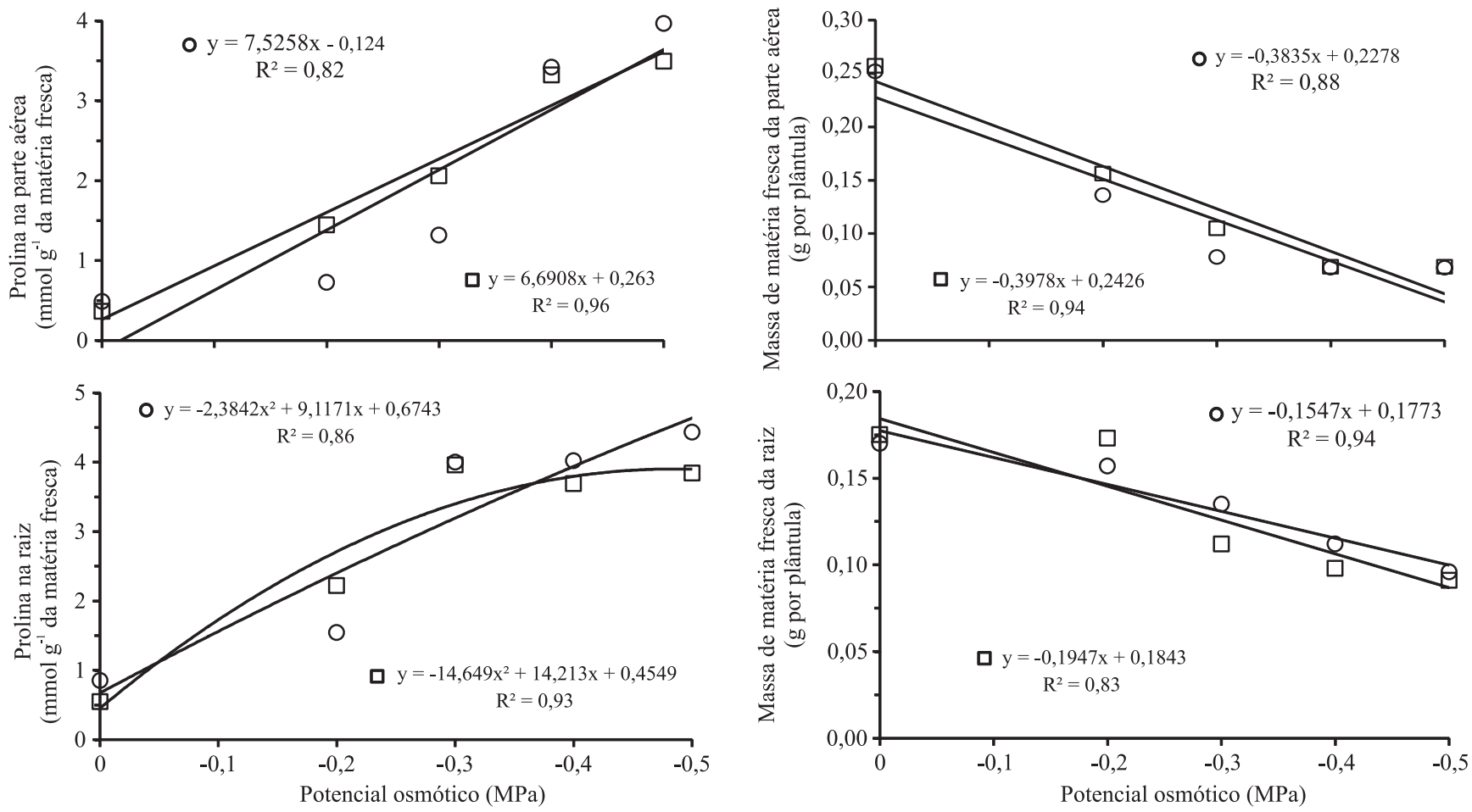

○ $0,0 \mathrm{mmol} \mathrm{L}^{-1}$ Putrescina $\quad$ 口 $0,5 \mathrm{mmol} \mathrm{L}^{-1}$ Putrescina

Figura 3. Teores de prolina ( $\mu \mathrm{mol} \mathrm{g} \mathrm{g}^{-1}$ da matéria fresca) e crescimento inicial (g por plântula) da parte aérea e de raízes primárias de plântulas de guandu (Cajanus cajan), submetidas ou não à aplicação de putrescina $\left(0,5 \mathrm{mmol} \mathrm{L}^{-1}\right)$, no experimento de restrição hídrica (PEG 6000). 
No tratamento com níveis de salinidade, a adição de putrescina exógena aumentou os teores de prolina na parte aérea e nas raízes das plântulas de guandu (Tabela 2). Quanto ao acúmulo de matéria seca nas raízes, em que houve interação dos efeitos da adição de putrescina com o de cultivares (Tabela 1), a poliamina aumentou a massa de matéria seca na cultivar BRS Mandarim, mas não produziu efeito na cultivar Caqui (Tabela 2).

No desdobramento dos efeitos da putrescina e da salinidade sobre o acúmulo de matéria seca na parte aérea e nas raízes, verificou-se que a putrescina exógena foi capaz de atenuar os efeitos da salinidade na redução do crescimento das raízes, mas apresentou efeito menos evidente na parte aérea (Figura 4).

A utilização da putrescina exógena em plantas de soja e citros submetidas a estresse salino aumenta os teores foliares de prolina e contribui para uma resposta positiva das plantas aos efeitos da salinidade (Su \& Bai, 2008; Sharma et al., 2011). No presente trabalho, entretanto, o tratamento com $0,5 \mathrm{mmol} \mathrm{L}^{-1} \mathrm{de}$ putrescina não foi efetivo em minimizar a redução do crescimento da parte aérea e das raízes das plântulas de guandu submetidas à restrição hídrica (Figura 3).

Possivelmente, a adição de $0,5 \mathrm{mmol} \mathrm{L^{-1 }}$ de putrescina não foi suficiente para promover a síntese de outras poliaminas endógenas que participam da atenuação dos estresses, como a espermidina e a espermina, uma vez que a putrescina é importante precursor da biossíntese dessas poliaminas, com reflexos positivos inclusive na produtividade das plantas (Quinet et al., 2010; Takahashi \& Kakehi, 2010). Estudos apontam incremento no crescimento de plântulas sob estresses com a aplicação de poliaminas (Khan et al., 2012) e redução de perdas hídricas por folhas de tabaco submetido à deficiência hídrica

Tabela 2. Teores de prolina na parte aérea e nas raízes primárias, e crescimento inicial das raízes de duas cultivares de guandu (Cajanus cajan) em função da adição de putrescina, no experimento sob estresse salino ${ }^{(1)}$.

\begin{tabular}{|c|c|c|c|c|}
\hline \multirow[t]{2}{*}{$\begin{array}{l}\text { Putrescina } \\
\left(\mathrm{mmol} \mathrm{L}^{-1}\right)\end{array}$} & \multicolumn{2}{|c|}{$\begin{array}{c}\text { Teor de prolina } \\
\left(\mu \mathrm{mol} \mathrm{g}{ }^{-1} \text { da matéria fresca) }\right.\end{array}$} & \multicolumn{2}{|c|}{$\begin{array}{l}\text { Massa de matéria seca } \\
\text { (g por plântula) }\end{array}$} \\
\hline & Parte aérea & Raízes primárias & BRS Mandarim & Caqui \\
\hline 0,0 & $96,0302 b$ & $124,7329 b$ & $0,1455 \mathrm{bB}$ & $0,1584 \mathrm{aA}$ \\
\hline 0,5 & $105,8915 \mathrm{a}$ & $142,5464 a$ & $0,1643 \mathrm{aA}$ & $0,1623 \mathrm{aA}$ \\
\hline
\end{tabular}

(Kotakis et al., 2014). Com 1 e $2 \mathrm{mmol} \mathrm{L}^{-1}$ de putrescina exógena, concentrações significativamente maiores que a usada no presente trabalho, têm-se observado aumento da massa seca de raízes de plântulas de romã (Punica granatum L.) sob restrição hídrica e estresse salino (Amri et al., 2011; Amri \& Mohammadi, 2012).

Nos casos em que não houve resposta à aplicação de putrescina, provavelmente, os potenciais osmóticos e as concentrações salinas foram severas a ponto de contrabalançar os possíveis efeitos atenuadores da putrescina. Outra possibilidade está relacionada à concentração utilizada dessa poliamida, que pode ter sido muito baixa. Além disso, constatou-se que ambas as cultivares de guandu utilizadas foram bastante suscetíveis aos tratamentos de restrição hídrica e salinidade.

Os resultados obtidos sugerem que os efeitos da putrescina exógena aplicada nas cultivares de guandu requerem outra abordagem investigativa que possa
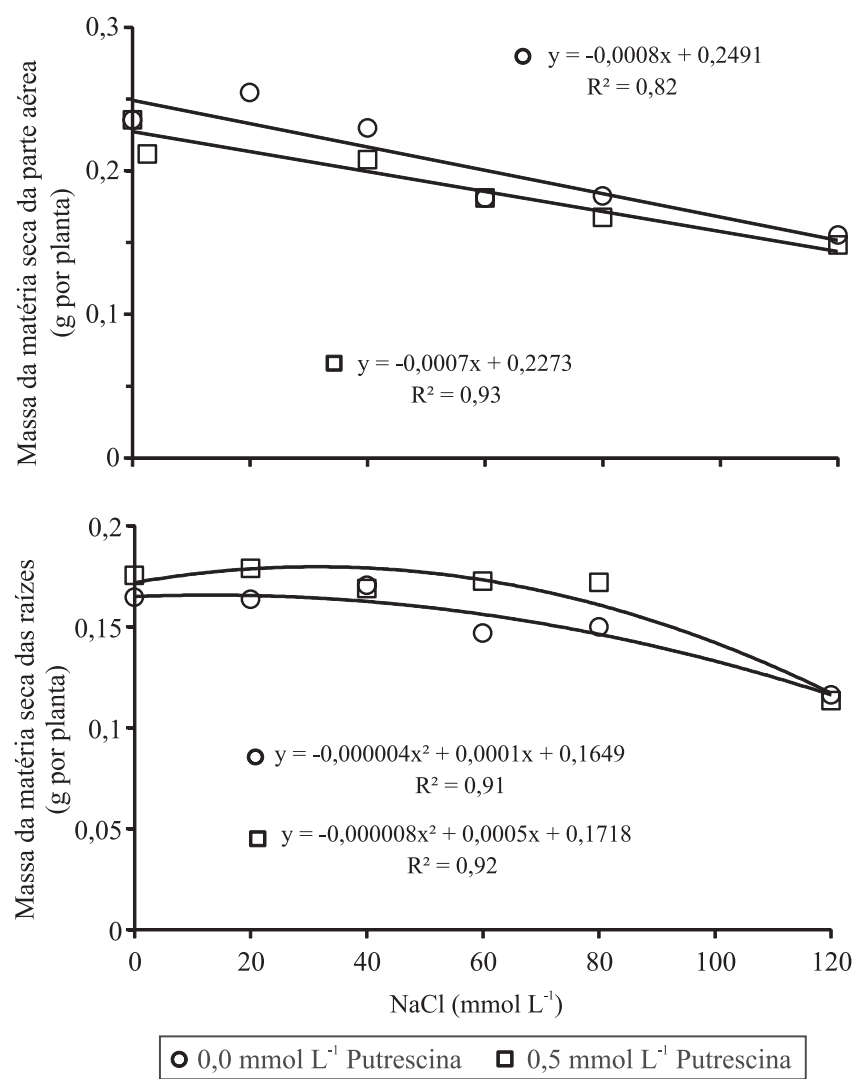

Figura 4. Crescimento inicial (g por plântula) da parte aérea e de raízes primárias de plântulas de guandu (Cajanus cajan), submetidas ou não à aplicação de putrescina $\left(0,5 \mathrm{mmol} \mathrm{L}^{1}\right)$, no experimento de estresse salino $(\mathrm{NaCl})$. 
revelar sua função no metabolismo das plântulas. $\mathrm{Su}$ \& Bai (2008), por exemplo, investigaram a atividade da enzima diamina oxidase, envolvida na degradação da putrescina e fornecedora de substratos para a síntese de prolina.

Apesar de não terem sido capazes de proteger as plântulas dos estresses estudados, os conteúdos de prolina podem ser considerados indicadores bioquímicos e fisiológicos efetivos dos efeitos da restrição hídrica e da salinidade, em guandu.

\section{Conclusões}

1. O crescimento das plântulas de guandu (Cajanus cajan) é acentuadamente reduzido pelas condições de restrição hídrica e salinidade.

2. Em ambas as cultivares, a putrescina exógena aumenta os teores de prolina em plântulas sob níveis moderados de deficiência hídrica e os reduz em níveis mais severos de estresse.

3. A putrescina exógena atenua os efeitos do estresse salino moderado sobre o crescimento das raízes das cultivares de guandu.

5. A prolina pode ser considerada um osmólito indicador bioquímico e fisiológico dos efeitos dos estresses hídricos e salinos em plântulas de guandu.

\section{Agradecimentos}

À Coordenação de Aperfeiçoamento de Pessoal de Nível Superior (Capes), pela concessão de bolsa de estudo; e à Fundação para o Desenvolvimento da Unesp (Fundunesp), pelo apoio financeiro.

\section{Referências}

ALCÁZAR, R.; PLANAS, J.; SAXENA, T.; ZARZA, X.; BORTOLOTTI,C.; CUEVAS, J.; BITRIÁN, M.; TIBURCIO, A.F.; ALTABELLA, Y. Putrescine accumulation confers drought tolerance in transgenic Arabidopsis plants overexpressing the homologous Arginine decarboxylase 2 gene. Plant Physiology and Biochemistry, v.48, p.547-552, 2010. DOI: $10.1016 / \mathrm{j}$. plaphy.2010.02.002.

AMRI, E.; MOHAMMADI, M.J. Effects of timing of drought stress on pomegranate seedlings (Punica granatum L. cv 〈Atabaki〉) to exogenous spermidine and putrescine polyamines. African Journal of Microbiology Research, v.6, p.5294-5300, 2012.

AMRI, E.; MOHAMMADI, M.; MORADI, M.; ZARE, K. The effects of spermidine and putrescine polyamines on growth of pomegranate (Punica granatum L. $\mathrm{cv}\langle$ Rabbab») in salinity circumstance. International Journal of Plant Physiology and Biochemistry, v.3, p.43-49, 2011.

ASHRAF, M.; AKRAM, N.A.; ALQURAINY, F.; FOOLAD, M.R. Drought tolerance: roles of organic osmolytes, growth regulators, and mineral nutrients. Advances in Agronomy, v.111, p.249-296, 2011. DOI: 10.1016/B978-0-12-387689-8.00002-3.

BATES, L.S.; WALDREN, R.P.; TEARE, I.D. Rapid determination of free proline for water stress studies. Plant and Soil, v.39, p.205207, 1973. DOI: 10.1007/BF00018060.

CVIKROVÁ, M.; GEMPERLOVÁ, L.; MARTINCOVÁ, O.; VANKOVÁ, R. Effect of drought and combined drought and heat stress on polyamine metabolism in prolineoverproducing tobacco plants. Plant Physiology and Biochemistry, v.73, p.7-15, 2013. DOI: 10.1016/j.plaphy.2013.08.005.

DUAN, J.; LI, J.; GUO, S.R.; KANG, Y. Exogenous spermidine affects polyamine metabolism in salinitystressed Cucumis sativus roots and enhances shortterm salinity tolerance. Journal of Plant Physiology, v.165, p.1620-1635, 2008. DOI: 10.1016/j. jplph.2007.11.006.

FARIDUDDIN, Q.; VARSHNEY, P.; YUSUF, M.; AHMAD, A. Polyamines: potent modulators of plant responses to stress. Journal of Plant Interactions, v.8, p.1-16, 2013. DOI: 10.1080/17429145.2012.716455.

FILIPPOU, P.; ANTONIOU, C.; FOTOPOULOS, V. The nitric oxide donor sodium nitroprusside regulates polyamine and proline metabolism in leaves of Medicago truncatula plants. Free Radical Biology and Medicine, v.56, p.172-183, 2013. DOI: 10.1016/j. freeradbiomed.2012.09.037.

FILIPPOU, P.; BOUCHAGIER, P.; SKOTTI, E.; FOTOPOULOS, V. Proline and reactive oxygen/nitrogen species metabolism is involved in the tolerant response of the invasive plant species Ailanthus altissima to drought and salinity. Environmental and Experimental Botany, v.97, p.1-10, 2014. DOI: 10.1016/j. envexpbot.2013.09.010

GORDIN, C.R.B.; MARQUES, R.F.; MASSETO, T.E.; SOUZA, L.C.F. de. Estresse salino na germinação de sementes e desenvolvimento de plântulas de niger (Guizotia abyssinica (L.f.) Cass.). Acta Botanica Brasilica, v.26, p.966-972, 2012. DOI: 10.1590/S0102-33062012000400026.

GUPTA, K.; DEY, A.; GUPTA, B. Plant polyamines in abiotic stress responses. Acta Physiologiae Plantarum, v.35, p.20152036, 2013. DOI: 10.1007/s11738-013-1239-4.

HUSSAIN, S.S.; ALI, M.; AHMAD, M.; SIDDIQUE, K.H.M. Polyamines: natural and engineered abiotic and biotic stress tolerance in plants. Biotechnology Advances, v.29, p.300311, 2011. DOI: 10.1016/j.biotechadv.2011.01.003.

JIANG, W.; BAI, B.; YANG, X.; YU, H.; LIU, Y. Exogenous application of abscisic acid, putrescine, or 2,4epibrassinolide at appropriate concentrations effectively alleviate damage to tomato seedlings from suboptimal temperature stress. Hortecnology, v.22, p.137-144, 2012.

KHAN, H.A.; ZIAF, K.; AMJAD, M.; IQBAL, Q. Exogenous application of polyamines improves germination and early seedling 
growth of hot pepper. Chilean Journal of Agricultural Research, v.72, p.429-433, 2012. DOI: 10.4067/S0718-58392012000300018.

KOTAKIS, C.; THEODOROPOULOU, E.; TASSIS, K.; OUSTAMANOLAKIS, C.; LOANNIDIS, N.E.; KOTZABASIS, K. Putrescine, a fastacting switch for tolerance against osmotic stress. Journal of Plant Physiology, v.171, p.48-51, 2014. DOI: 10.1016/j.jplph.2013.09.015.

KUMAR, R.R.; KARJOL, K.; NAIK, G.R. Variation of sensitivity to drought stress in pigeon pea (Cajanus cajan [L.] Millsp) cultivars during seed germination and early seedling growtH. World Journal of Science and Technology, v.1, p.11-18, 2011.

LUTTS, S.; HAUSMAN, J.F.; QUINET, M.; LEFÈVRE, I. Polyamines and their roles in the alleviation of ion toxicities in plants. In: AHMAD, P.; AZOOZ, M.M.; PRASAD, M.N.V. (Ed.). Ecophysiology and responses of plants under salt stress. New York: Springer, 2013. p.315-353. DOI: 10.1007/978-1-4614-47474_12.

MACHADO NETO, N.B.; CUSTÓDIO, C.C.; COSTA, P.R.; DONA, F.L. Restrição hídrica induzida por diferentes agentes osmóticos na germinação e vigor de sementes de feijão. Revista Brasileira de Sementes, v.28, p.142-148, 2006. DOI: 10.1590/ S0101-31222006000100020.

MAIA, J.M.; FERREIRASILVA, S.L.; VOIGT, E.L.; MACÊDO, C.E.C. de; PONTE, L.F.A.; SILVEIRA, J.A.G. Atividade de enzimas antioxidantes e inibição do crescimento radicular de feijão caupi sob diferentes níveis de salinidade. Acta Botanica Brasilica, v.26, p.342-349, 2012

MARIJUAN, M.P.; BOSCH, S.M. Ecophysiology of invasive plants: osmotic adjustment and antioxidants. Trends in Plant Science, v.18, p.660-666, 2013. DOI: 10.1016/j.tplants.2013.08.006.

MUNNS, R. Plant adaptations to salt and water stress: differences and commonalities. In: TURKAN, I (Ed.). Plant responses to drought and salinity stress: developments in a postgenomic Era. London: Elsevier, 2011. p.132. (Advances in botanical research, $57)$.
QUINET,M.;NDAYIRAGIJE,A.;LEFÈVRE,I.;LAMBILLOTTE, B.; DUPONTGILLAIN, C.C.; LUTTS, S. Putrescine differently influences the effect of salt stress on polyamine metabolism and ethylene synthesis in rice cultivars differing in salt resistance. Journal of Experimental Botany, v.61, p.2719-2733, 2010. DOI: $10.1093 / \mathrm{jxb} / \mathrm{erq} 118$.

SHARMA, D.K.; DUBEY, A.K.; SRIVASTAV, M.; SINGH, A.K.; SAIRAM, R.K.; PANDEY, R.N.; DAHUJA, A.; KAUR, C. Effect of putrescine and paclobutrazol on growth, physiochemical parameters, and nutrient acquisition of salt-sensitive citrus rootstock Karna khatta (Citrus karna Raf.) under $\mathrm{NaCl}$ stress. Journal of Plant Growth Regulation, v.30, p.301-311, 2011. DOI: 10.1007/s00344-011-9192-1.

SU, G.X.; BAI, X. Contribution of putrescine degradation to proline accumulation in soybean leaves under salinity. Biologia Plantarum, v.52, p.796-799, 2008. DOI: 10.1007/s10535-0080156-7.

SZÁBADOS, L.; KOVACS, H.; ZILBERSTEIN, A.; BOUCHEREAU, A. Plants in extreme environments: importance of protective compounds in stress tolerance. TURKAN, I (Ed.). Plant responses to drought and salinity stress: developments in a postgenomic Era. London: Elsevier, 2011. p.105-150. (Advances in botanical research, 57). DOI: 10.1016/B978-0-12-3876928.00004-7.

TAKAHASHI, T.; KAKEHI, J.I. Polyamines: ubiquitous polycations with unique roles in growth and stress responses. Annals of Botany, v.105, p.1-6, 2010. DOI: 10.1093/aob/mcp259.

TROVATO, M.; MATTIOLI, R.; COSTANTINO, P. Multiple roles of proline in plant stress tolerance and development. Rendiconti Lincei, v.19, p.325-346, 2008. DOI: 10.1007/s12210-008-0022-8.

VERBRUGGEN, N.; HERMANS, C. Proline accumulation in plants: a review. Amino Acids, v.35, p.753-759, 2008. DOI: 10.1007/s00726-008-0061-6.

WILHELM, C. Salt stress resistance-multisite regulation in focus. Journal of Plant Physiology, v.171, p.1, 2014. DOI: 10.1016/j. jplph.2013.11.001.

Recebido em 18 de fevereiro de 2013 e aprovado em 27 de dezembro de 2013

Pesq. agropec. bras., Brasília, 49, n.1, p.18-25, jan. 2014 DOI: 10.1590/S0100-204X2014000100003 